\title{
MiR-376a and Histone Deacetylation 9 Form A Regulatory Circuitry in Hepatocellular Carcinoma
}

\author{
Yongxia Zheng ${ }^{\mathrm{a}}$ Huan Chen ${ }^{\mathrm{b}}$ Manxiang Yin ${ }^{c}$ Xiaoqian Ye $\mathrm{Guiqian}^{\mathrm{C}} \mathrm{Chen}^{\mathrm{a}}$ \\ Xinmei Zhou ${ }^{\mathrm{a}}$ Lei Yin ${ }^{\mathrm{d}}$ Chengwen Zhang $^{\mathrm{a}}$ Baoyue Ding ${ }^{\mathrm{a}}$ \\ aMedical College, Jiaxing University, Jiaxing, ${ }^{b}$ State Key Laboratory of Proteomics, Beijing Proteome \\ Research Center, Beijing Institute of Radiation Medicine, Beijing, 'Pathology Department, Hospital \\ of Zhejiang General Corps of Armed Police Forces, Jiaxing, 'Eastern Hepatobiliary Surgery Hospital, \\ Second Military Medical University, Shanghai, China
}

\section{Key Words}

MiR-376a • HDAC9 • Hepatocellular Carcinoma

\begin{abstract}
Background/Aims: Our previous study has demonstrated that down-regulation of miR-376a might contribute to the development of hepatocellular carcinoma (HCC), but the mechanism underlying this down-regulation remains obscure. Methods/ Results: histone deacetylase (HDAC) inhibitor increased the level of miR-376a in L02 and Huh7 cells by up-regulating the acetylation level of histone 3 at the Maternally expressed 3 (Meg3) differentially methylated region (DMR). Interestingly, HDAC9, a histone deacetylase responsible for deacetylating lysine 18 of histone 3 (H3K18), was identified as the target of miR-376a. In addition, HDAC9 siRNA increased the expression of miR-376a by up-regulating the global histone H3K18 acetylation level, with Meg3 DMR included. Finally, miR-376a and HDAC9 were inversely correlated in HCC. Conclusion: HDAC9 plays an important role both as effects and targets of miR376 .
\end{abstract}

Z. Yongxia and C. Huan contributed equally to this work.

Zheng Yongxia,

and Ding Baoyue
Medical College, Jiaxing University, \#118 Jiahang Road, Jiaxing 314001, ZJ, (China)

E-Mail zhengyongxia@163.com, E-Mail lena_310@163.com

\section{KARGER 125}




\section{Introduction}

Hepatocellular carcinoma (HCC) is the sixth most prevalent cancer worldwide [1]. Although many studies have described multiple genetic and epigenetic changes in HCC, the current evidence cannot explain the full complexity of HCC [2]. Our previous study demonstrated that miR-376a suppressed the proliferation and induced the apoptosis of HCC cells [3], but little is known about the underlying mechanism of its deregulation in HCC.

The MiR-376 family is clustered together within the imprinted Dlk1-Dio3 region on human 14q32 [4], and imprinting of the Dlk1-Dio3 cluster is regulated by DNA methylation and histone acetylation at differentially methylated regions (DMRs) [5, 6]. Knowing that epigenetic alteration silences the 14q32 miRNA cluster in epithelial ovarian cancer [7], we hypothesized that epigenetic modification might be responsible for the down-regulation of miR-376a in HCC.

Histone deacetylases (HDAC) are commonly dysregulated in cancer including HCC [8, 9]. Several studies have provided evidence about the role of miRNA as both targets and effects of histone acetylation [10-13]. HDAC9 as a class II HDAC family member is not only responsible for epigenetic modification but a candidate target of miR-376a. In this study, we focus on the mechanism of miR-376a regulation by HDAC9 in HCC. Our data suggests that HDAC9-mediated epigenetic modification may contribute to the down-regulation of the miR-376 cluster in HCC.

\section{Material and Methods}

Clinical materials

Forty-one cancerous and adjacent noncancerous specimens were obtained from patients with informed consent who underwent surgery for primary HCC at the Eastern Hepatobiliary Surgery Hospital (Shanghai, China) between 2010 and 2011. The specimens were frozen in liquid nitrogen immediately after surgical resection and preserved at $-80^{\circ} \mathrm{C}$. Both cancerous and noncancerous specimens were histologically confirmed, and all HCC tumors originated from HBV infection. The study was approved by the Institute Research Ethics Committee of the Eastern Hepatobiliary Surgery Hospital.

Cell culture and transfection

L02 and Huh7 cell lines (Institute of Biochemistry and Cell Biology, Shanghai, China) were cultured in DMEM (Biowest, Loire, France) with 10\% fetal bovine serum (FBS, Biowest, Loire, France) in a humidified atmosphere containing $5 \% \mathrm{CO}_{2}$ at $37^{\circ} \mathrm{C}$. MiR-376a mimics, negative control RNA, siRNA-HDAC9 (4463nt, Gene Bank accession no. NM_001204144) and control siRNA were obtained from GenePharma (Shanghai, China). RNA sequences are listed in Table S1. Transfection was performed using Lipofectamine 2000 (Invitrogen, CA, USA) according to the manufacturer's protocols.

\section{RT-PCR}

Total RNA was isolated from the prepared liver samples and cells using TRIzol reagent (Invitrogen, Carlsbad, CA, USA). cDNA was synthesized following the manufacturer' protocols (MBI Fermentas, Vilnius, Lithuania). qRT-PCR was performed with a standard SYBR-green PCR kit (TOYOBO, Osaka, Japan), and genespecific PCR amplification was performed using the ABI 7300 (Applied Biosystems, Darmstadt, Germany). qRT-PCR reactions were performed in triplicate. Relative gene expression was calculated with the $2^{-\Delta \Delta C t}$ method after normalization to the expression of GAPDH or U6. The primers are listed in Table 1.

\section{Western blotting analysis}

Tissues and cells were lysed in RIPA lysis buffer (Beyotime, Jiangsu, China). The lysates were centrifuged at $12,000 \mathrm{rpm}$ at $4^{\circ} \mathrm{C}$ for $10 \mathrm{~min}$. Equal amounts of protein were separated using $10-15 \%$ sodium dodecyl sulfonate-polyacrylamide gel electrophoresis and transferred to nitrocellulose membranes (Boguang, Shanghai, China) subsequently. For immunodetection, the membranes were respectively incubated with antibodies specific for HDAC9 (Epitomics, Burlingame, CA), H3K18ac (Abcam, Cambridge, 


\begin{tabular}{rl|l} 
Cellular Physiology & \multicolumn{1}{c}{ Cell Physiol Biochem 2015;35:729-739 } \\
\cline { 2 - 3 } and Biochemistry & $\begin{array}{l}\text { DOI: 10.1159/000369733 } \\
\text { Publisned onIne: January 30, 2015 }\end{array}$ & $\begin{array}{l}\text { O 2015 S. Karger AG, Basel } \\
\text { www.karger.com/cpb }\end{array}$ \\
\cline { 2 - 3 } & Zheng et al.: The Reciprocal Modulation Between miR-376a and HDAC9
\end{tabular}

Table 1. Primers and Nucleotides

\begin{tabular}{|c|c|c|}
\hline Name & & Sequences \\
\hline \multirow{2}{*}{ miR-376a } & RT & 5'-GTCGTATCCAGTGCAGGGTCCGAGGTATTCGCACTGGATAC GACACGTGG-3' \\
\hline & Reverse & 5'- ATCATAGAGGAAAATCCACG -3' \\
\hline \multirow{2}{*}{ U6 } & Forward & 5'- CTCGCTTCGGCAGCACA -3' \\
\hline & Reverse & 5'- AACGCTTCACGAATTTGCGT-3' \\
\hline \multirow{2}{*}{ MiR-376b } & RT & 5'-GTCGTATCCAGTGCAGGGTCCGAGGTATTCGCACTGGATACGACAACATG-3' \\
\hline & Reverse & 5'-ATCATAGAGGAAAATCCATGTTG-3' \\
\hline \multirow{2}{*}{ Meg3 DMR } & Forward & 5'-CATGTTG CCCCCTTTGCCACTTAG-3' \\
\hline & Reverse & 5'-CCCAGCAACCCGGAGTGTACTGTG-3' \\
\hline \multirow{2}{*}{ MiR-376c } & RT & 5'-GTCGTATCCAGTGCAGGGTCCGAGGTATTCGCACTGGATACGACTGGAGA-3' \\
\hline & Reverse & 5'-GGTGGATATTCCTTCTATGTT-3' \\
\hline \multirow{2}{*}{ MiR-494 } & $\mathrm{RT}$ & 5'-GTCGTATCCAGTGCAGGGTCCGAGGTATTCGCACTGGATACGACGAGGTT-3' \\
\hline & Reverse & 5'-TGAAACATACACGGGAAACCTCT-3' \\
\hline \multicolumn{2}{|l|}{ MiRNA universal sense } & 5'-GTGCAGGGTCCGAGGT-3' \\
\hline HDAC9 & Forward & 5'-GCCCACCACACATCATTGG-3' \\
\hline 3'-UTR & Reverse & 5'-AATGTGTACTTGTAGGATGGAGATGTTC-3' \\
\hline \multirow{2}{*}{ Mu-HDAC9 3'-UTR } & Forward & 5'-TGACGCACGTGCCCTTTAAAAACAG-3' \\
\hline & Reverse & 5'-TTGATTTATTGTCTAAGAAA-3' \\
\hline \multirow{2}{*}{ GAPDH-RT } & Forward & 5'- AGAAGGCTGGGGCTCATTTG -3' \\
\hline & Reverse & 5'-AGGGGCCATCCACAGTCTTC-3' \\
\hline \multirow{2}{*}{ HDAC9-RT } & Sense & 5'-AGCCCATCTCACCTTTAGACC -3' \\
\hline & Anti-sense & 5' - ATTGCTTCTCACGGACAACAG -3' \\
\hline MiR-376a & Sense & 5'-AUCAUAGAGGAAAAUCCACGU-3' \\
\hline mimics & Anti-sense & 5'-GUGGAUUUUCCUCUAUGAUUU -3' \\
\hline Negative & Sense & 5'-UUCUCCGAACGUGUCACGUTT -3' \\
\hline control & Anti-sense & 5'-ACGUGACACGUUCGGAGAATT -3' \\
\hline HDAC9 & Forward & 5'-GGACAGACCUCAGGAUGAU-3' \\
\hline siRNA & Reverse & 5'-AUCAUCCUGAGGUCUGUCC-3' \\
\hline LNA miR-376a probe & Anti-sense & 5'-ACGTGGATTTTCCTCTATGAT-3' \\
\hline
\end{tabular}

UK) and GAPDH (Epitomics, Burlingame, CA). The immunoblotting sample was incubated with horseradish peroxidase (HRP)-coupled anti-rabbit secondary antibodies (ProteinTech, Chicago, USA) and visualized using enhanced chemiluminescence (Pierce, Rockford, USA)

\section{Immunohistochemistry}

The cancerous and adjacent non-cancerous tissue blocks were sliced into $5 \mu \mathrm{m}$ sections, then fixed with formalin and embedded with paraffin. Samples were first incubated with an anti-HDAC9 antibody (Epitomics, Burlingame, CA), and then incubated with a biotinylated secondary antibody using the ChemMate Envision Kit (K5001, DAKO, Hamburg, Germany). All stained sections were examined under a light microscope at magnification of 200x. Staining density was analyzed using Image-Pro Plus 5 (Media Cybernetics, Bethesda, MD).

In situ hybridization

In situ hybridization was performed with an ISH kit for paraffin embedded sections (Boster, Wuhan, China). Briefly, a total of 3 pmol digoxigenin-labeled locked nucleic acid probes (Huirui, Shanghai, China) were diluted into $200 \mu \mathrm{l}$ hybridization buffer and allowed to hybridize on the slides at $37^{\circ} \mathrm{C}$ overnight. The slides were then washed with $2 \times \mathrm{SSC}$ solution at $37^{\circ} \mathrm{C}$ and incubated with biotinylated mouse antidigoxigenin antibody at $4^{\circ} \mathrm{C}$ overnight. Following incubation with strept avidin-biotin complex (SABC) and biotin-conjugated horseradish peroxidase, horseradish peroxidase reaction was carried out with DAB staining solution for $30 \mathrm{~min}$. The sequence of the LNA probe is listed in Table 1.

Luciferase reporter constructs, site-directed mutagenesis and luciferase reporter assay.

The 3'UTR of HDAC9 containing the miR-376a response element was cloned into the pGL4.13 luciferase reporter vector (Promega, Wisconsin Madison, USA) between the Xba I and Fse I restriction sites using a directional RT-PCR cloning strategy. Mutant 3'UTR of HDAC9 was synthesized by PCR. The primers are listed in Table S1. The resulting luciferase reporter constructs pGL-HDAC9-UTR and pGL-HDAC9-MuKARGER 
UTR containing the wild-type 3'UTR and mutant 3'UTR of HDAC9 were sequenced to ensure accuracy. Huh7 cells were seeded in a 24-well plate and transfected with $200 \mathrm{ng}$ luciferase reporter constructs with miR376a mimics or NC. Each sample was co-transfected with $20 \mathrm{ng}$ renilla luciferase control vector pGL4.70 (Promega, Wisconsin Madison, USA) to monitor transfection efficiency. The pGL4.13 (Promega, Wisconsin Madison, USA) control vector was used as a control. After $48 \mathrm{~h}$, all protein extracts were analyzed using dual luciferase reporter system (Promega, Wisconsin Madison, USA).

\section{5-Aza-CdR and PBA treatment}

5-Aza-CdR (Sigma-Aldrich, St. Louis, MO) and PBA (Sigma-AldrichSt, St. Louis, MO) were dissolved in PBS and DMSO at a concentration of $3 \mathrm{mM}$ and $3 \mathrm{M}$, respectively. L02 and Huh7 cells were seeded at $2 \times 10^{5}$ cells per well in 6-well plates, and subsequently treated with 5-Aza-CdR ( $3 \mu \mathrm{M})$ and PBA (3mM) for $24 \mathrm{~h}$. After removing 5-Aza-CdR, PBA administration was continued for 5 days with the medium containing PBA replaced every $24 \mathrm{~h}$. DMSO-treated cells were used as controls. Each experiment was repeated at least three times.

\section{Chromatin immunoprecipitation (ChIP)}

ChIP was performed on 5-Aza-CdR and PBA treated Huh7 or L02 cells, and HDAC9 siRNA (4463nt, Gene Bank accession no. NM_001204144) treated Huh7 or L02 cells using the ChIP Assay Kit (Millipore, Billerica, MA, USA) with ChIP-grade antibodies against either for acetyl-histone H3 (Abcam, Cambridge, UK) or acetyl-histone H3K18 (Abcam, Cambridge, UK). The ChIP-enriched DNAs were quantitated using qPCR with primers targeting Meg3-DMR, and normalized against the respective input DNAs.

\section{Statistics}

Statistical analysis was performed by using the SPSS statistics software package (SPSS). All results were expressed as mean $\pm \mathrm{SD}$, and $\mathrm{P}<0.05$ was used for significance.

\section{Results}

miR-376a is frequently down-regulated in HCC by epigenetic modification

It was found in our previous study [3] that miR-376a was frequently down-regulated in human HCC specimens, but the underlying mechanism remains obscure. Knowing that epigenetic alterations can deregulate Dlk1-Dio3 microRNA cluster in epithelial ovarian cancer [7], we wondered whether it was also responsible for the dysregulation of miR-376a in HCC. Therefore, we measured the level of miR-376a after treatment of the normal liver cell line (L02) and HCC cell line (Huh7) with the DNA methyltransferase (DNMT) inhibitor 5-aza-2'- deoxycytidine (5-Aza-CdR) and the histone deacetylase (HDAC) inhibitor 4-phenylbutyric acid (PBA) [7]. As shown in Fig. 1 (A and B), miR-376a was up-regulated by 15.6 \pm 2.14

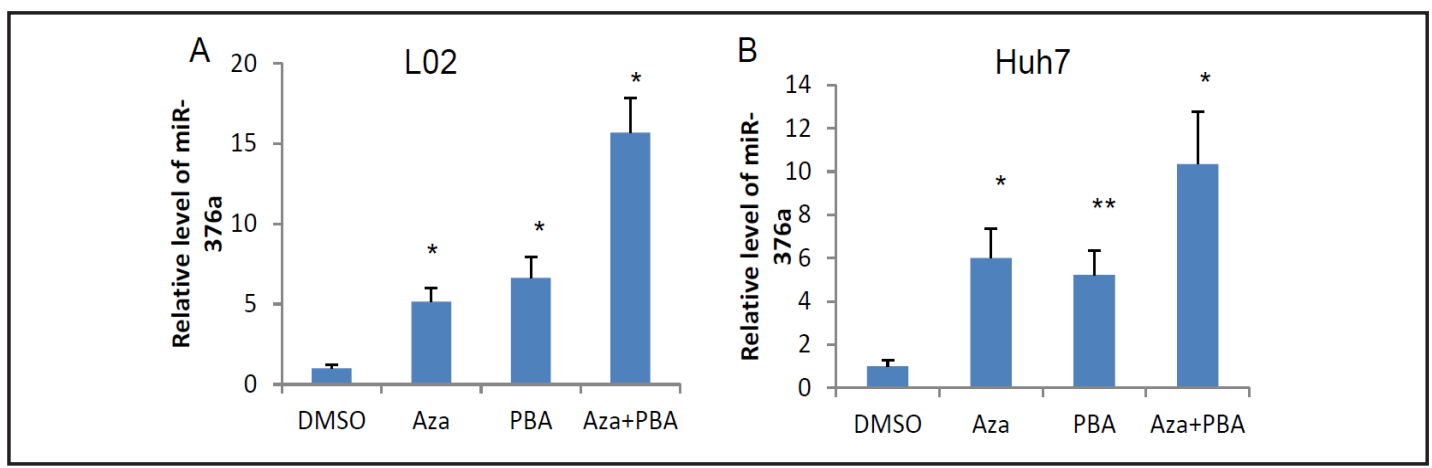

Fig. 1. miR-376a is highly induced by 5-Aza-CdR and PBA treatment. L02 (A) and Huh7 (B) cells were treated with 5-Aza-CdR and PBA, and the miR-376a expression level was analyzed by qRT-PCR. U6 RNA expression was used as a loading control. Data from three independent experiments are shown as the means \pm SD and analyzed by paired sample test. $\left({ }^{*} \mathrm{p}<0.05,{ }^{* *} \mathrm{p}<0.01\right)$. 


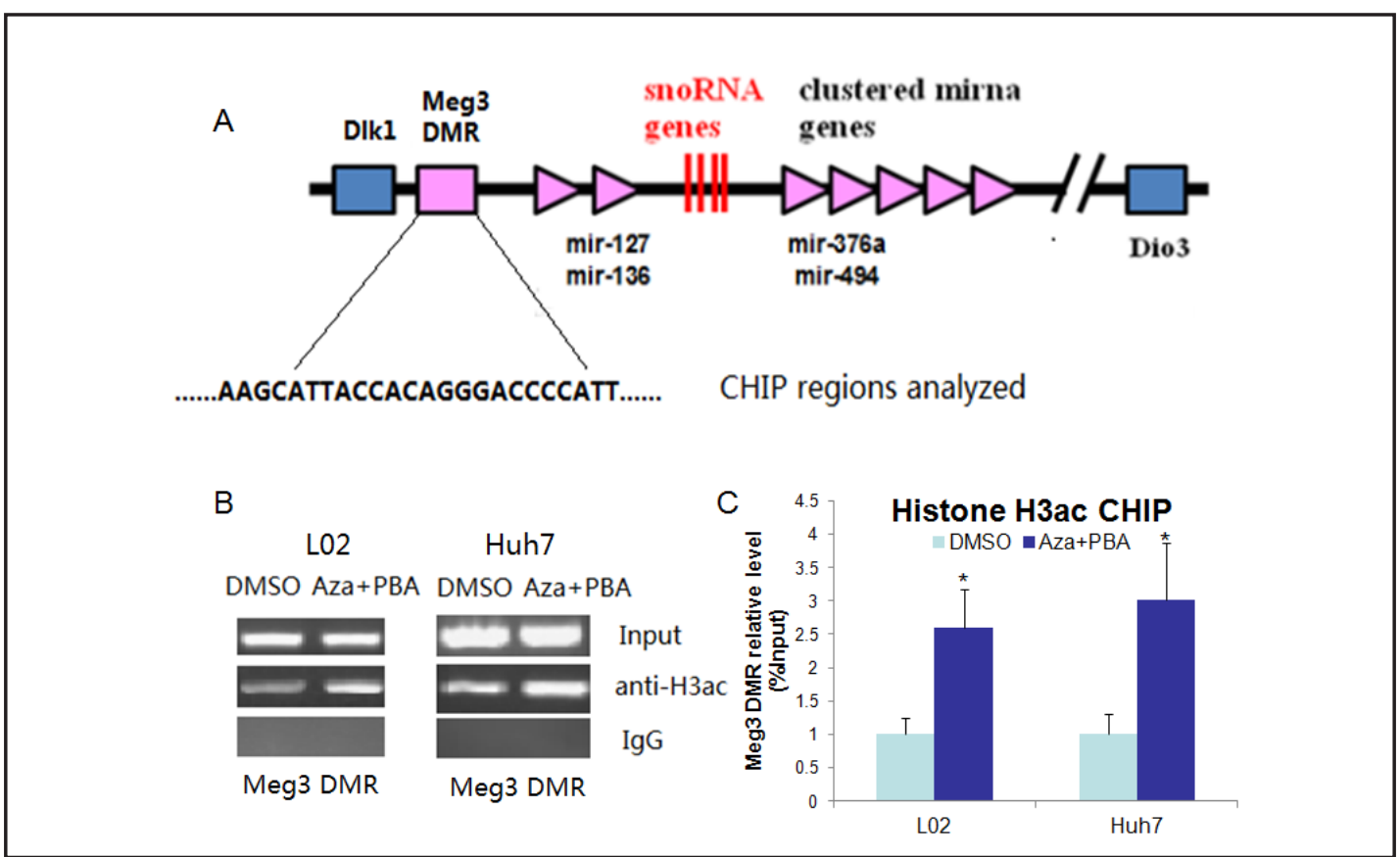

Fig. 2. The histone acetylation level located in the Meg3 DMR is upregulated in response to 5-Aza-CdR and PBA. A. Schematic representation of the Dlk1-Dio3 gene cluster with Meg3 DMR. B and C. 5-Aza-CdR and PBA treatment increases the histone 3 acetylation level of Meg3 DMR. ChIP derived DNA was amplified by qRT-PCR using specific primers. The levels of qPCR products are expressed as a percentage of input DNA. Data are presented as the means \pm SD and analyzed by paired sample $t$ test, based on at least three independent experiments. $\left({ }^{*} \mathrm{p}<0.05,{ }^{* *} \mathrm{p}<0.01\right)$.

fold $(\mathrm{p}=0.010)$ after the combination treatment with 5-Aza-CdR and PBA, and by $6.6 \pm 1.3$ fold ( $p=0.025)$ after treatment with PBA alone in L02 cells. In addition, miR-376a was upregulated by $10.35 \pm 2.4$ fold $(\mathrm{p}=0.013$ ) after the combination treatment with 5 -Aza-CdR and PBA, and by $5.21 \pm 1.12$ fold $(\mathrm{p}=0.009)$ after treatment with PBA alone in Huh7 cells. These findings suggest that miR-376a was regulated by epigenetic modification in hepatocytes and hepatocarcinoma cells.

The histone acetylation level located in the Meg3 DMR is up-regulated in response to 5-Aza-CdR and PBA

To further investigate the mechanism of miR-376a regulation by HDACi, the acetylation level of histone located in the Meg3 DMR was detected (Fig. 2A), knowing that it is mainly responsible for the epigenetic regulation of Dlk1-Dio3 cluster [6]. More specifically, we performed CHIP-PCR assays to determine whether up-regulation of miR-376a in response to 5-Aza-CdR and PBA was due to the increased acetylation level of histone 3 at the Meg3-DMR. As shown in Fig. 2 (B and C), acetyl-histone 3 was up-regulated by $3.01 \pm 0.85$ fold ( $p=0.018$ ) in 5-Aza-CdR and PBA treated Huh7 cells, and by $2.59 \pm 0.58$ fold ( $\mathrm{p}=0.042$ ) in 5-Aza-CdR and PBA treated L02 cells. These data suggest that the increased acetylation level of histone 3 at the Meg3 DMR was partially responsible for the up-regulation of miR-376a in response to HDACi.

miR-376a down-regulates HDAC9 directly

miRNAs play an important role in epigenetic modification by targeting epigenetic factors [14-17]. Interestingly, histone deacetylasd 9 (HDAC9) was also found to be the candidate target of miR-376a (Fig. 3A). To determine whether miR-376a target HDAC9 in HCC cells, we examined the expression of HDAC9 in miR-376a treated Huh7 or L02 cells. As shown in 

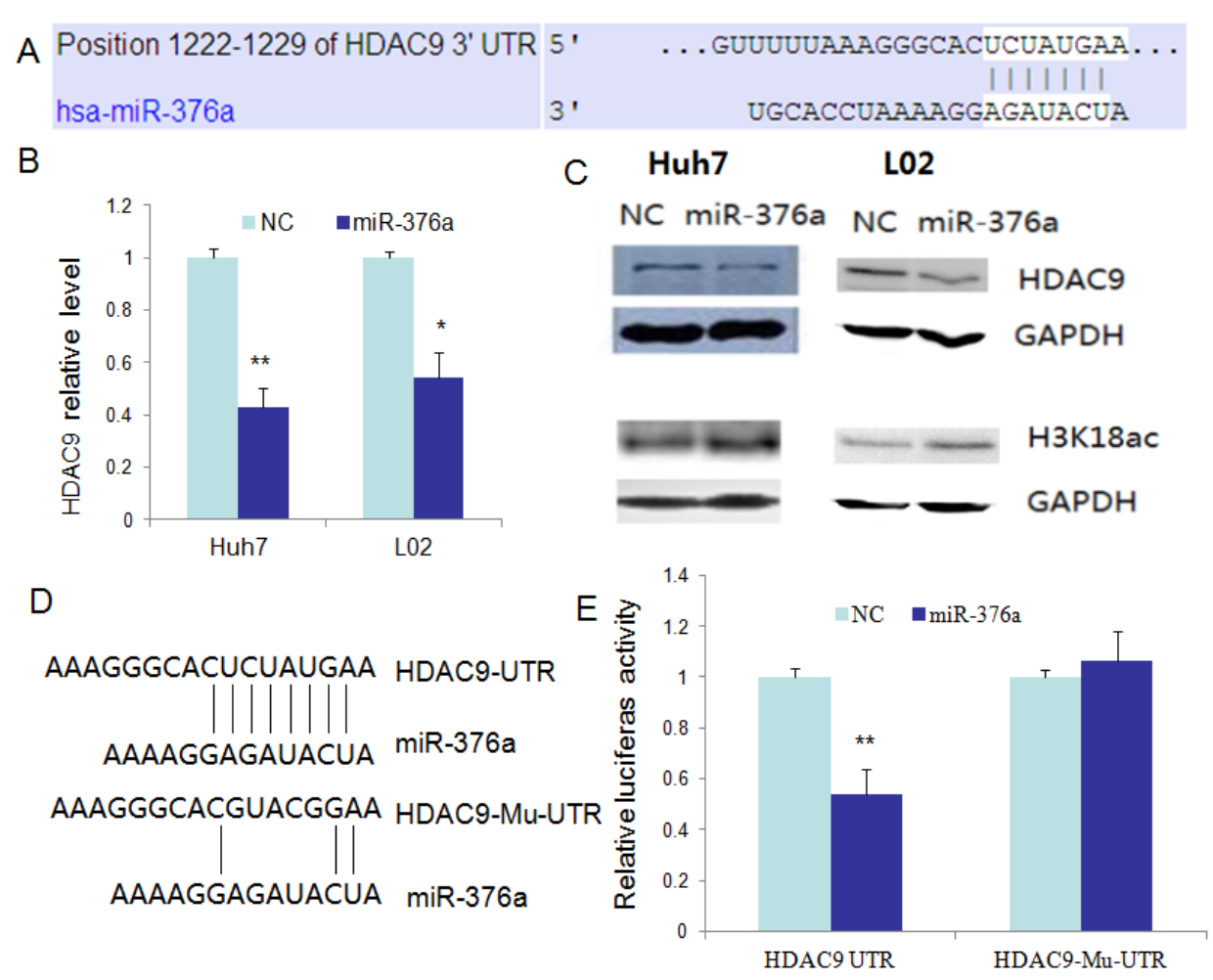

Fig. 3. miR-376a down-regulates HDAC9 directly. A. Graphic overview of the binding site of miR-376a in the 3'UTR sequences of HDAC9. B and C. HDAC9 was analyzed by qRT-PCR and Western blotting analysis after Huh7 or L02 cells were transfected with miR-376a mimics. GAPDH was used as a control. D. miR-376a binding site in the 3'UTR (top) and mutated sites in 3'UTR (bottom) of HDAC9 constructed in a luciferase system. E. The repression of HDAC9 gene mediated by the 3'UTR was analyzed by luciferase reporter assay. Data from three independent experiments are shown as the means $\pm S D$ and analyzed by paired sample t test. $\left({ }^{*} \mathrm{p}<0.05,{ }^{* *} \mathrm{p}<0.01\right)$.

Fig. 3 (B and C), miR-376a not only decreased the mRNA level of HDAC9 in Huh7 (43\% $7 \%$, $\mathrm{p}=0.004)$ and $\mathrm{L} 02(54 \% \pm 9 \%, \mathrm{p}=0.014)$ cells but decreased the protein level of HDAC9. To determine whether HDAC9 was inhibited by miR-376a directly, the 3'UTR region of HDAC9 was fused to a luciferase reporter (pGL 4.13). As shown in Fig. 3E, miR-376a repressed the luciferase activity of the wild type plasmid (HDAC9-UTR) but not the mutant plasmid (HDAC9-mut-UTR), indicating that HDAC9 was a direct target of miR-376a.

Knowing that HDAC9 can increase site-specific lysine histone acetylation at H3 (H3K9, H3K14, and H3K18) globally [18] and H3K9ac and H3K14ac can be deacetylated by multiple HDACs, we selected H3K18 as the downstream effector of HDAC9. To test whether miR-376a could regulate the acetyl-histone H3K18 level by targeting HDAC9, we examined the acetylhistone H3K18 level in miR-376a treated Huh7 or L02 cells. It was found that miR-376a not only inhibited HDAC9 but increased the acetyl-histone H3K18 level in the two cell types (Fig. 3C). These results indicate that miR-376a could induce up-regulation of global acetylhistone H3K18 by targeting HDAC9.

HDAC9 inhibits the expression of miR-376a through down-regulating the H3K18ac level at Meg3 DMR

Knowing that miR-376a was induced by HDACi, it was reasonable to assume that HDAC9 may repress the expression of miR-376a. Then, We knocked-down HDAC9 by transfecting HDAC9 siRNA into Huh7 or L02 cells, using siRNA as the negative control. After 
A

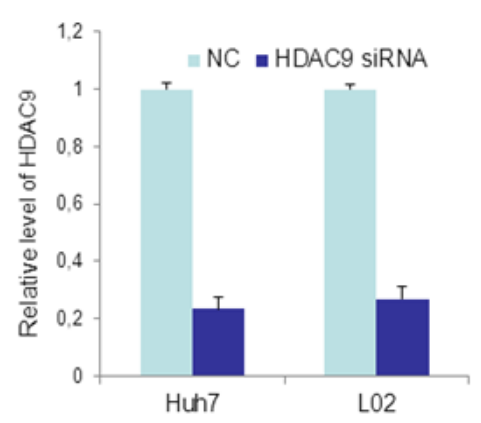

C

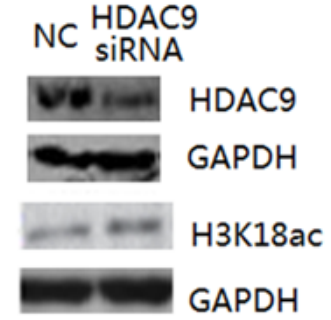

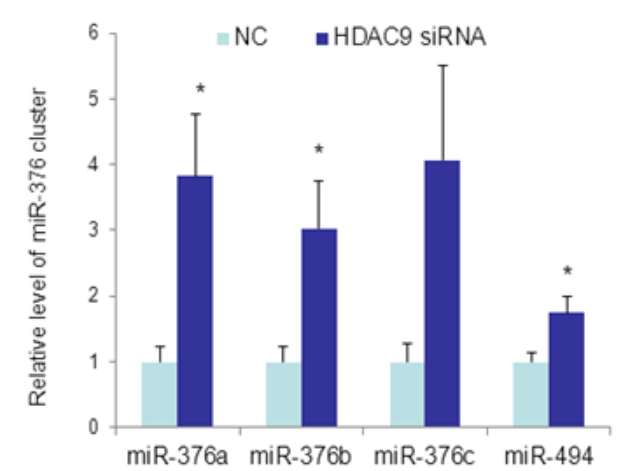

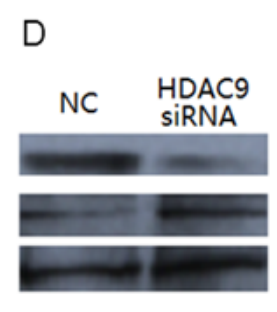

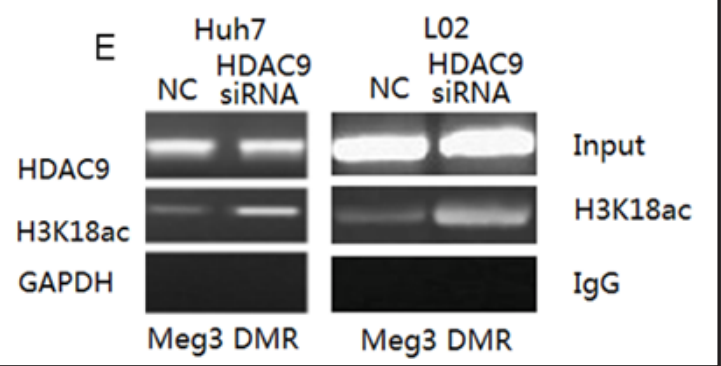

Fig. 4. HDAC9 inhibits the expression of the miR-376a cluster by up-regulating the acetylation level of histone H3K18. A. Identification of HDAC9 knockdown efficiency by siRNA via qRT-PCR. GAPDH was used as fold control. B. miR-376a cluster, including miR-376a, miR-376b, miR-376c and miR-494, was analyzed by qRT-PCR after Huh7 cells were treated with HDAC9 siRNA. Data from three independent experiments are shown as the means \pm SD and analyzed by paired sample t test. $\left(* p<0.05,{ }^{* *} p<0.01\right)$. C and D. HDAC9 and acetyl-histone H3K18 protein levels were analyzed by Western blotting analysis after Huh7 or L02 cells were treated with HDAC9 siRNA. E. HDAC9 siRNA increases the histone H3K18 acetylation level of MEG3 DMR both in Huh7 and L02 cells. CHIP derived DNA was amplified by qRT-PCR using specific primers.

48-h transfection, the transfection efficiency was confirmed by qPCR and Western Blotting analysis by measuring the miR-376a level. As shown in Fig. 4 (B and C), HDAC9 was reduced markedly by HDAC9 siRNA both at mRNA level $(23 \% \pm 7 \%, p=0.003)$ and protein level. Additionally, HDAC9 increased miR-376a level by $3.83 \pm 0.93$ fold $(\mathrm{p}=0.046)$, suggesting that HDAC9 repressed the expression of miR-376a. Except for miR-376a, the Dlk1-Dio3 cluster also contained miR-376b, miR-376c and miR-494 (Fig. 4A). Therefore, the expression of these miRNAs was detected using the same method. It was found that HDAC9 siRNA increased the level of miR-376b, miR-376c and miR-494 by $3.02 \pm 0.72$ fold ( $p=0.027$ ), 4.06 \pm 0.03 fold $(p=0.03)$ and $1.74 \pm 0.24$ fold $(p=0.049)$, respectively. These results indicate that HDAC9 inhibited the expression of the miR-376a family (Fig. 4D).

To explore the underlying mechanism of how HDAC9 inhibited the miR-376a family, the total H3K18ac level was examined by Western blotting, and the histone H3K18ac level at Meg3 DMR was detected by CHIP-PCR. It was found that HDAC9 siRNA increased the histone H3K18 acetylation level both globally (Fig. 4B, C) and at the Meg3 DMR (Fig. 4E) in Huh7 or L02 cells, suggesting that HDAC9 may repress miR-376a by means of acetylating histone H3K18 at Meg3 DMR.

HDAC9 expression is inversely correlated with miR-376a in human HCC specimens

To determine the status of HDAC9 in HCC, we detected its mRNA level in 41 HCC specimens by qPCR. As shown in Fig. 5A, HDAC9 and miR-376a levels were inversely expressed in the HCC samples. In addition, miR-376a was stained by in situ hybridization using an anti-miR-376a LNA probe, and HDAC9 and acetyl-histone H3K18 were analyzed by immunohistochemistry. As shown in Fig. 5 (B, C and D), miR-376a and acetyl-histone 
Fig. 5. HDAC9 is upregulated, while H3K18ac is down-regulated in human HCC tissues compared with paired adjacent noncancerous hepatic tissues. (A) Expression of miR-376a and HDAC9 shows an inverse correlation in HCC. HDAC9 mRNA expression was analyzed by qRTPCR. GAPDH was used as fold control. Statistical analysis was performed using pearson's correlation coefficient $(\mathrm{n}=41)$. (B) Immunohistochemistry staining of HDAC9 in HCC. (C) Immunohistochemistry staining of acetyl-histone H3K18 in HCC. Representative immunohisto- chemical stainings in HCC with noncancerous tissues $(\mathrm{N})$ or cancerous tissues (T) shown. (D) In situ hybridization of miR-376a in paraffin embedded HCC sections.

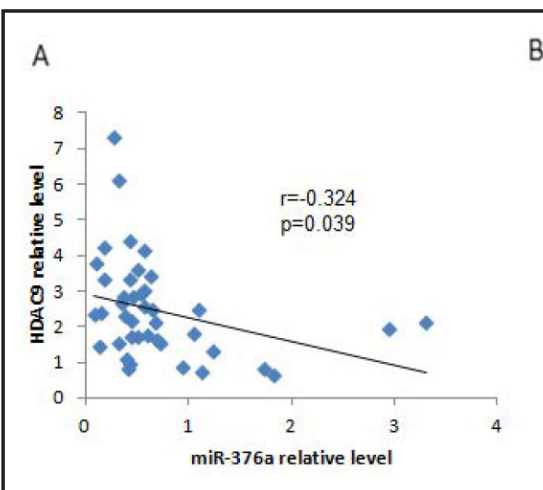

C

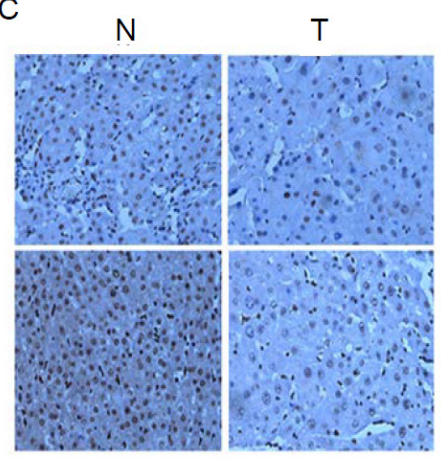

H3K18ac

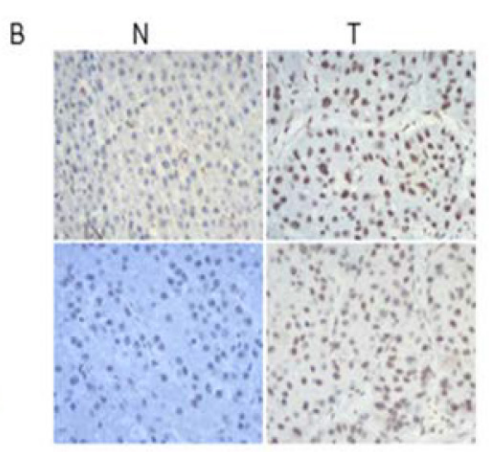

HDAC9

$\mathrm{D}$

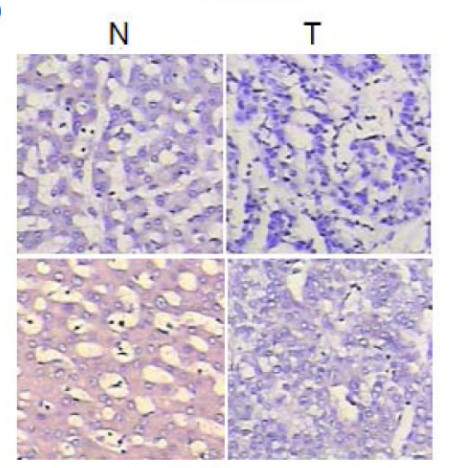

miR-376a

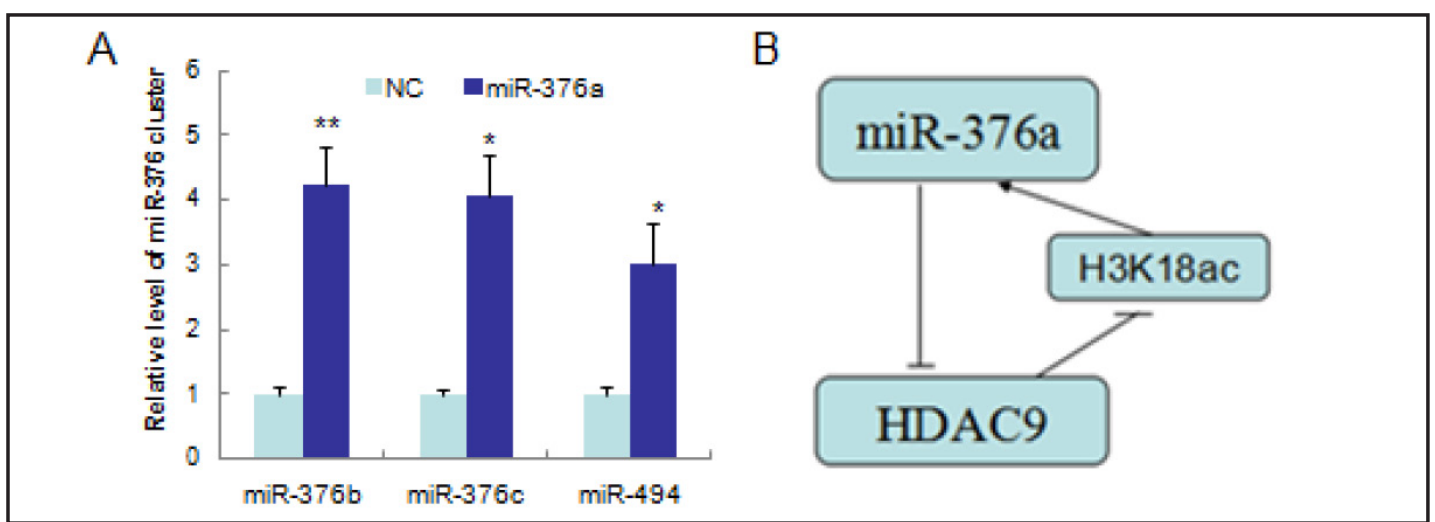

Fig. 6. Autoregulation of the miR-376a. (A) miR-376a increases the level of miR-376b, miR-376c and miR494. qRT-PCR was used to detect the level of miR-376b, miR-376c and miR-494, using U6 as fold control. Data from three independent experiments are shown as the means \pm SD and analyzed by paired sample $t$ test. ( ${ }^{*} \mathrm{p}<0.05,{ }^{* *} \mathrm{p}<0.01$ ). (B) A model depicting miR-376a auto-regulation. MiR-376a directly suppresses HDAC9 level, while HDAC9 inhibits the expression of miR-376 cluster via down-regulating the acetyl-histone H3K18.

H3K18 were decreased, while HDAC9 was clearly over-expressed in the cancerous samples as compared with the adjacent non-cancerous samples. Taken together, these observations suggest that HDAC9 was inversely correlated with miR-376a in human HCC specimens.

The autoregulatory effect of miR-376a

Knowing that HDAC9 and miR-376a could inhibit each other, we wondered whether miR-376a could activate itself through the HDAC9/miR-376a pathway. To separate the KARGER 
endogenous miR-376a from exogenous miR-376a mimics, the miR-376 family and miR-494 were detected. As shown in Fig. 6, miR-376a increased the level of miR-376b, miR-376c and miR-494 by $4.24 \pm 0.56$ fold ( $p=0.009$ ), $4.06 \pm 0.65$ fold ( $p=0.013$ ) and $3.01 \pm 0.62$ fold $(p=0.04$ ), respectively, suggesting that miR-376a may auto-regulate itself via the miR-376a/HDAC9 pathway.

\section{Discussion}

HDACs are enzymes involved in chromatin remodeling by deacetylating the lysine residues of histone, and play a pivotal role in epigenetic regulation [19]. Dysregulation of HDACs in cancers has been well documented in many studies and more HDAC inhibitors have been attempted in clinical trials for the treatment of various malignancies [20-23]. In addition, accumulating evidence suggests that microRNAs are involved in dysregulation of HDACs. For instance, Sun et al ${ }^{[12]}$ reported that the HDAC4/SP1/ miR-200a regulatory network contributed to aberrant histone acetylation in HCC. Another study [24] reported that HDAC9 transcriptionally repressed the miR17-92 cluster and promoted angiogenesis. It was found in this study that HDAC9 was up-regulated in HCC specimens, which is consistent with the previous study of Fisher et al. [25] and Zheng et al. [26], and miR-376a could modulate the epigenetic modification by targeting HDAC9.

In this study, we demonstrated that HDAC9 inhibited miR-376a by decreasing the acetyl-histone H3K18 level at Meg3-DMR. Beyond that, other factors may also participate in the inhibition of HDAC9 on miR-376a. For instance, HDAC9 was reported to repress MEF2 mediated transcription [27], and MEF2 was reported to be responsible for the transcription of miR379-410 cluster [28]. However, whether MEF2 mediates the inhibition of HDAC9 on miR-376a remains unanswered at present, and the epigenetic mechanism may only partially explain how HDAC9 inhibit miR-376a.

Additionally, we demonstrated that miR-376a and HDAC9 comprised a positive feedback loop, which is reported to be an ubiquitous signal transduction motif that allows systems to convert graded inputs into decisive, all-or-none outputs [29, 30]. Therefore, this positive feedback loop may result in continuous decrease of miR-376a in HCC and selfreinforcing proliferation caused by the down-regulation of miR-376a. Thus, it is presumable that this feed-forward loop may play a role in the process of chronic inflammation to cancer initiation.

In summary, our findings reveal a regulatory circuitry between miR-376a and HDAC9 in HCC and suggest that HDAC9, a direct target of miR-376a, mediated silencing of miR-376 cluster by epigenetic alterations.

\section{Abbreviations}

HDAC9 (histone deacetylase 9); HCC (hepatocellular carcinoma); Meg3 (Maternally expressed 3); H3K18 (lysine 18 of histone 3); DMR (differentially methylated region. Dlk1Dio3 region, genomic region marked by Dlk1 gene and Dio3 gene); 5-Aza-CdR (5-aza-2'deoxycytidine); PBA (4-phenyl-butyric acid); CHIP (Chromatin immuno- precipitation); H3K9ac (histone 3 acetyl lysine 9); H3K14ac (histone 3 acetyl lysine 14); MEF2 (myocyte enhancer factor-2).

\section{Disclosure Statement}

The authors declare no competing financial interests. 
Zheng et al.: The Reciprocal Modulation Between miR-376a and HDAC9

\section{Acknowledgments}

This study was supported by Grant Y201330031 from Zhejiang Provincial Education Department; Grant 81400654, 81201809, 81400489 from the National Science Foundation of China; and Grant LQ12H30005 from Zhejiang Provincial Natural Science Foundation; and Grant of the 12th Five-year Plan for University Key Academic Subject (Pharmacology), Zhejiang Province, China.

\section{References}

$>_{1}$

Forner A, Llovet JM, Bruix J: Hepatocellular carcinoma. Lancet 2012;379:1245-1255.

Pan H, Fu X, Huang W: Molecular mechanism of liver cancer. Anticancer Agents Med Chem 2011;11:493499.

3 Zheng Y, Yin L, Chen H, Yang S, Pan C, Lu S, Miao M, Jiao B: miR-376a suppresses proliferation and induces apoptosis in hepatocellular carcinoma. FEBS Lett 2012;586:2396-2403.

-4 Seitz H, Royo H, Bortolin ML, Lin SP, Ferquson-Smith AC, Cavaille J: A Large Imprinted microRNA Gene Cluster at the Mouse Dlk1-Gtl2 Domain. Genome Res 2004;14:1741-1748.

-5 Stadtfeld M, Apostolou E, Akutsu H, Fukuda A, Follett P, Natesan S, Kono T, Shida T, Hochedlinger K: Aberrant silencing of imprinted genes on chromosome 12qF1 in mouse induced pluripotent stem cells. Nature 2010;465:175-181.

-6 Mcmurray EN, Schmidt JV: Identification of imprinting regulators at the meg3 differentially methylated region. Genomics 2012;100:184-194.

7 Zhang L, Volinia S, Bonome T, Calin GA, Greshock J, Yang N, Liu CG, Ginnakakis A, Alexious P, Haseqawa K, Johnstone CN, Megraw MS, Adams S, Lassus H, Huang J, Kaur S, Liang S, Sethupathy P, Leminen A, Simossis VA, Sandaltzopoulos R, Naomoto Y, Katsaros D, Gimotty PA, Demichele A, Huang Q Butzow R, Rustqi AK, Weber BL, Birrer MJ, Hatzigeorqiou AG, Corce CM, Coukos G: Genomic and epigenetic alterations deregulate microRNA expression in human epithelial ovarian cancer. Proc Natl Acad Sci USA 2008;105:7004-7009.

8 Witt O, Deubzer HE, Milde T, Oehme I: HDAC family: What are the cancer relevant targets? Cancer Lett 2009;277:8-21.

-9 Bai X, Wu L, Liang T, Liu Z, Li J, Li D, Xie H, Yin S, Yu J, Lin Q, Zheng S: Overexpression of myocyte enhancer factor 2 and histone hyperacetylation in hepatocellular carcinoma. J Cancer Res Clin Oncol 2008;134:8391.

10 Roccaro AM, Sacco A, Jia X, Azab AK, Maiso P, Ngo HT, Azab F, Runnels J, Quang P, Ghobrial IM: microRNAdependent modulation of histone acetylation in Waldenstrom macroglobulinemia. Blood 2010;116:15061514.

- 11 Liu S, Wu LC, Pang J, Santhanam R, Schiwind S, Wu YZ, Hickey CJ, Becher H, Maharry K, Radmacher MD, Li C, Whitman SP, Mishra A, Stauffer N, Eiring AM, Briesewitz R, Baiocchi RA, Chan KK, Paschka P, Caligiuri MA, Byrd JC, Corce CM, Bloomfield CD, Perrotti D, Garzon R, Marcucci G: Sp1/NFkB/HADC/miR-29b regulatory network in KIT-driven myeloid leukemia. Cancer Cell 2010;17:333-347.

12 Yuan JH, Yang F, Chen BF, Lu Z, Huo XS, Zhou WP, Wang F, Sun SH: The Histone Deacetylase 4/Sp1/miR200a Regulatory Network Contributes to Aberrant Histone Acetylation in Hepatocellular Carcinoma. Hepatology 2011;54:2025-2035.

13 Noonan EJ, Place RF, Pookot D, Basak S, Whitson JM, Hirata H, Giardina C, Dahiya R: miR-449a targets HDAC-1 and induces growth arrest in prostate cancer. Oncogene 2009;28:1714-1724.

-14 Kumar R, Xi Y: microRNA, epigenetic machinery and lung cancer. Thoracic Cancer 2011;2:35-44.

15 Xu Y, Guo Y, Liu L, Cai R, Qian C: The Reciprocal Modulation Between Epigenetic and microRNA and The Application for Treatment of Malignant Tumors. Progress in Biochemistry and Biophysis 2008;35:13431350.

16 Liep J, Rabien A, Jung K: Feedback networks between microRNAs and epigenetic modifications in urological tumors. Epigenetics 2012;7:315-325.

17 Maruyama R, Suzuki H, Yamamoto E, Imai K, Shinomura Y: Emerging links between epigenetic alterations and dysregulation of noncoding RNAs in cancer. Tumor Biol 2012;33:277-285. 


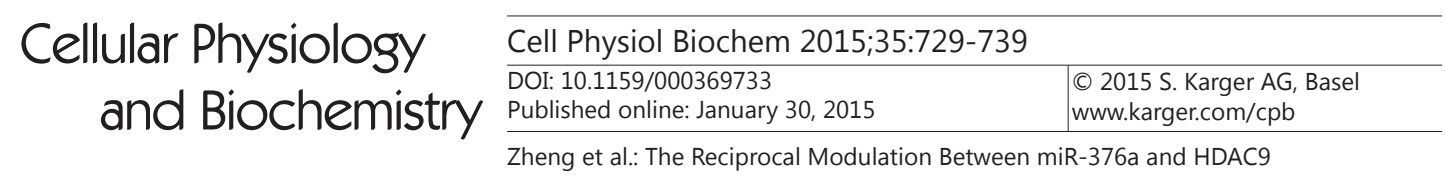

18 Yan K, Cao Q, Reilly CM, Young NL, Garcia BA, Mishra N: Histone deacetylase 9 deficiency protects against effector T cell-mediated systemic autoimmunity. J Biol Chem 2011;286:28833-28843.

19 Bertos NR, Wang AH, Yang XJ. Class II histone deacetylases: structure, function and regulation. Biochem Cell Biol 2001;79:243-252.

-20 Lachenmayer A, Toffanin S, Cabellos L, Alsinet C, Hoshida Y, Villanueva A, Minquez B, Tsai HW, Ward SC, Thung S, Friedman SL, Lovet JM: Combination therapy for hepatocellu- lar carcinoma: additive preclinical efficacy of the HDAC inhibitor panobinostat with sorafenib. J Hepatol 2012;56:1343-1350.

21 Komatsu N, Kawamata N, Takeuchi S, Yin D, Chien W, Miller CW, Koeffler HP: SAHA, a HDAC inhibitor, has profound anti-growth activity against non-small cell lung cancer cells. Oncol Rep 2006;15:187-191.

-22 Platta CS, Greenblatt DY, Kunnimalaiyaan M, Chen H: The HDAC inhibitor trichostatin A inhibits growth of small cell lung cancer cells. J Surg Res 2007;142:219-226.

23 Rikkishi H: Autophagic and apoptotic effects of HDAC inhibitors on cancer cells. J Biomed Biotechnol 2011;830260.

24 Kaluza D, Kroll J, Gesierich S, Manavski Y, Boeckel JN, Doebele C, Zelent A, Rossiq L, Zeiher AM, Augustin HG, Urbich C, Dimmeler S: Histone deacetylase 9 promotes angiogenesis by targeting the antiangiogenic miRNA17-92 cluster in endothelial cells. Arterioscler Thromb Vasc Biol 2013;33:533-543.

25 Archer KJ, Mas VR, Maluf DG, Fisher RA: High-throught assessment of CpG site methylation for distinguishing between HCV-cirrhosis and HCV-associated hepatocellular carcinoma. Mol Genet Genomics 2010;283:341-349. Bai X, Wu L, Liang T, Liu Z, Li J, Li D, Xie H, Yin S, Yu J, Lin Q Zheng S: Overexpression of myocyte enhancer factor 2 and histone hyperacetylation in hepatocellular carcinoma. J Cancer Res Clin Oncol 2008;134:8391.

27 Petrie K, Guidez F, Howell L, Healy L, Waxman S, Greaves M, Zelent A: The histone deacetylase 9 gene encodes multiple protein isoforms. J Biol Chem 2003;278:16059-16072.

-28 Fiore R, Khudayberdiev S, Christensen M, Siegel G, Flavell SW, Kim TK, Greenberg ME, Schratt G: Mef2mediated transcription of the miR379-410 cluster regulates activity-dependent dendritogenesis by finetuning Pumilio2 protein levels. EMBO J 2009;28:697-710.

29 Brandman O, Ferrell JE Jr, Li R, Meyer T: Interlinked fast and slow positive feedback loops drive reliable cell decisions. Science 2005;310:496-498.

30 Kobayashi T, Chen L, Aihara K: Modeling genetic swithes with positive feedback circuits. J Theor Biol 2003;221:379-399. 